\title{
REPRESENTATION OF FOURIER INTEGRALS AS SUMS. II
}

\section{R. J. DUFFIN}

Let the functions $f(x)$ and $g(x)$ be defined in terms of the function $\phi(x)$ by the sums:

$$
f(x)=\sum_{1}^{\infty} \frac{\alpha_{n}}{n} \phi\left(\frac{x}{n}\right)
$$

and

$$
g(x)=\sum_{1}^{\infty} \frac{\alpha_{n}}{x} \phi\left(\frac{n}{x}\right) .
$$

Here $\alpha_{n}=\sin (\pi n / 2), n=1,2, \cdots$.

In a previous paper [1 $]^{1}$ it was shown that under quite weak restrictions on $\phi$ :

$$
\begin{aligned}
& g(x)=\int_{0}^{\infty} \operatorname{sn} x t f(t) d t \\
& f(x)=\int_{0} \operatorname{sn} x t g(t) d t .
\end{aligned}
$$

Here $\operatorname{sn} x$ is an abbreviation for $\sin (\pi x / 2)$.

This paper gives other conditions for the validity of these identities. The previous conditions permitted $\phi$ to have various types of discontinuity. The present paper is concerned with smooth functions; however, the growth at 0 and $\infty$ is permitted to be greater than before. Theorems 1, 2, and 3 of the previous paper together with Theorems 2 and 3 of this paper form a fairly complete elementary theory of these identities. The proofs given here do not depend on the previous paper.

The results of this paper hinge on the possibility of defining the Fourier sine transform for functions which do not vanish at infinity. Theorem 1 below shows that this is possible merely by employing summability. It is to be noted that Theorem 1 is not true, as it stands, for the cosine transform. For example, the cosine transform of any constant evaluated by such a definition would vanish. Hence the inversion formula could not apply. A theory of "generalized Fourier

Presented to the Society December 30, 1948; received by the editors November 16, 1948.

${ }^{1}$ Numbers in brackets refer to the references cited at the end of the paper. 
integrals" has been developed by Fry, Hahn, Wiener, Burkill, Bochner, and others [2]. These writers do not differentiate between sine and cosine transforms, and it appears that their results contain no equivalent of Theorem 1.

THEOREM 1. Let $f(x)$ be a function whose derivative $f^{\prime}(x)$ is of bounded variation in the interval $(0, \infty)$, and let $f^{\prime}(x)$ vanish at $\infty$. Then the integral (3) is summable $C_{1}$ to define a function $g(x)$. The integral (4) is improperly integrable at 0 and gives $f(x)$ for $x>0$.

Proof. A function which satisfies the conditions of the Theorem and also vanishes at 0 will be said to satisfy Conditions $B$. It is quite easy to see that the theorem is true for $f(x)$ a constant, so it is sufficient to prove the theorem under Conditions B. Two integrations by parts show that

$$
\int_{0}^{k} \sin t f(t) d t=-\cos k f(k)+\sin k f^{\prime}(k)-\int_{0}^{k} \sin t d f^{\prime}(t) .
$$

The integral on the right converges absolutely as $k \rightarrow \infty$. Therefore the integral on the left is summable $C_{1}$ provided the first two terms on the right are summable. But

$$
\frac{1}{h} \int_{0}^{h} \cos k f(k) d k=\frac{\sin h}{h} \int_{0}^{h} f^{\prime}(k) d k-\frac{1}{h} \int_{0}^{h} \sin k f^{\prime}(k) d k .
$$

Because $f^{\prime}$ vanishes at $\infty$ it follows that the Cesaro mean of the term $\cos k f(k)$ vanishes as $h \rightarrow \infty$. For the same reason the Cesaro mean of the second term on the right in (5) vanishes. Thus for $x>0$

$$
\int_{0}^{\infty} \operatorname{sn} x t f(t) d t=-\frac{4}{\pi^{2} x^{2}} \int_{0}^{\infty} \operatorname{sn} x t d f^{\prime}(t) .
$$

It is worth noting that a simpler type of summability will also establish (6). It is sufficient to take the limit in (5) for $k=\pi \nu / 2 ; \nu=1$, $3,5, \cdots$. This causes the undesirable term $\cos k f(k)$ to drop out.

Denote the function defined by (6) as $g(x)$, and let $b$ and $c$ be positive constants such that $b \geqq 1 \geqq c$. Then

$$
\int_{c}^{b} \operatorname{sn} x g(x) d x=-\frac{4}{\pi^{2}} \int_{0}^{\infty} \int_{0}^{b} \frac{\operatorname{sn} x \operatorname{sn} x t}{x^{2}} d x d f^{\prime}(t) .
$$

The inner integral on the right may be written

$$
\int_{1}^{b} \frac{\operatorname{sn} x \operatorname{sn} x t}{x^{2}} d x+\int_{c}^{1} \frac{\operatorname{sn} x-\pi x / 2}{x^{2}} \operatorname{sn} x t d x+\int_{c}^{1} \frac{\pi \operatorname{sn} x t}{2 x} d x .
$$


It is obvious that the first two integrals converge uniformly in $t$ as $b \rightarrow \infty$ and $c \rightarrow 0$. It is easy to show that the third integral converges uniformly for $t$ in any finite range and that for all $t$ it is uniformly bounded. Thus relation (7) is valid for $b=\infty$ and $c=0$ provided the integral on the left is evaluated as an improper integral at the lower limit. As is well known

$$
\frac{4}{\pi^{2}} \int_{0}^{\infty} \frac{\operatorname{sn} x \operatorname{sn} x t}{x^{2}} d x= \begin{cases}t ; & 0 \leqq t \leqq 1, \\ 1 ; & t \geqq 1 .\end{cases}
$$

Substituting (8) in (7) gives $f(1)=\int_{0}^{\infty}$ sn $x g(x) d x$. The observation that $f(k x)$ for $k>0$ satisfies the Conditions $\mathrm{B}$ and has the transform $g(x / k) / k$ clearly completes the proof.

THEOREM 2. Let $\phi(x)$ be differentiable for $x \geqq 1$. Let $\phi^{\prime}(x)$ be of bounded variation in the interval $(1, \infty)$, and let $\phi^{\prime}(x)$ vanish at $\infty$. Let the same conditions be satisfied by $\Phi(x)=\phi(1 / x) / x$. Then for $x>0$ the series (1) and (2) are summable $C_{1}$ and satisfy (3) and (4). The integrals are summable $C_{1}$ at $\infty$ and improperly integrable at 0 .

Proof. Consider first the special case when $\phi(x)$ satisfies Conditions $B$. Let a function $f^{\prime}(x)$ be defined by the series

$$
f^{\prime}(x)=\sum_{1}^{\infty} \frac{\alpha_{n}}{n^{2}} \phi^{\prime}\left(\frac{x}{n}\right) \text {. }
$$

The function $\phi^{\prime}$ is bounded, so the series is uniformly convergent and may be integrated termwise. Hence

$$
\begin{aligned}
\int_{0}^{x} f^{\prime}(x) d x & =\sum_{1}^{\infty} \frac{\alpha_{n}}{n^{2}} \int_{0}^{x} \phi^{\prime}\left(\frac{x}{n}\right) d x \\
& =\sum_{1}^{\infty} \frac{\alpha_{n}}{n} \phi\left(\frac{x}{n}\right) .
\end{aligned}
$$

Thus the series (1) converges and defines a function $f(x)$ whose derivative $f^{\prime}(x)$ is given by (9). From (10) it is seen that $f(x)$ vanishes at 0 . From (9) it is seen that $f^{\prime}(x)$ vanishes at $\infty$. Let $V$ denote the operation of taking the variation on the interval $(0, \infty)$. Then from (9)

$$
V f^{\prime}(x) \leqq \sum_{1}^{\infty} \frac{1}{n^{2}} V \phi^{\prime}\left(\frac{x}{n}\right) \leqq 2 V \phi^{\prime}(x) .
$$

From these considerations it follows that $f(x)$ defined by (1) satisfies Conditions B if $\phi(x)$ does.

Let $f_{m}^{\prime}(x)$ denote the first $m$ terms of the series (9). Then 


$$
\begin{aligned}
\int_{0}^{\infty} \operatorname{sn} t d f_{m}^{\prime}(t) & =\sum_{1}^{m} \frac{\alpha_{n}}{n^{2}} \int_{0}^{\infty} \operatorname{sn} t d \phi^{\prime}\left(\frac{t}{n}\right) \\
& =\int_{0}^{\infty} \sum_{1}^{m} \frac{\alpha_{n}}{n^{2}} \operatorname{sn} n t d \phi^{\prime}(t) .
\end{aligned}
$$

From (11) it is clear that $V\left(f^{\prime}-f_{m}^{\prime}\right) \rightarrow 0$ as $m \rightarrow \infty$; hence the integral on the left in (12) approaches $\int_{0}^{\infty} \mathrm{sn} t d f^{\prime}(t)$. The series on the right converges uniformly. Thus

$$
\frac{4}{\pi^{2}} \int_{0}^{\infty} \operatorname{sn} t d f^{\prime}(t)=\int_{0}^{\infty} \sigma(t) d \phi^{\prime}(t) .
$$

Here $\sigma(t)=\sum_{1}^{\infty}\left(4 \alpha_{n} /(\pi n)^{2}\right)$ sn $n t$ is a well known series:

$$
\sigma(t)=t / 2,-1 \leqq t \leqq 1 ; \sigma(t)=(2-t) / 2,1 \leqq t \leqq 3 ; \text { and so on. }
$$

Thus

$$
\begin{aligned}
-2 \int_{0}^{5} \sigma(t) d \phi^{\prime}(t)+\phi^{\prime}(5) & =2 \int_{0}^{5} \phi^{\prime}(t) d \sigma(t) \\
& =\int_{0}^{1} \phi^{\prime}(t) d t-\int_{1}^{3} \phi^{\prime}(t) d t+\int_{3}^{5} \phi^{\prime}(t) d t \\
& =\phi(1)-[\phi(3)-\phi(1)]+[\phi(5)-\phi(3)] .
\end{aligned}
$$

But $\phi^{\prime}(x)$ vanishes at $\infty$, so in general

$$
-\int_{0}^{\infty} \sigma(t) d \phi^{\prime}(t)=\lim \sum_{1}^{\nu} \alpha_{n} \phi(n)-\alpha_{\nu} \phi(\nu) / 2 ; \quad \nu=1,3,5, \cdots .
$$

It is easy to show that a series summable by the method indicated in (14) is also summable by Cesaro means when use is made of the fact that $\phi(\nu) / \nu \rightarrow 0$.

Combining (6), (13), and (14) gives

$$
\int_{0}^{\infty} \operatorname{sn} t f(t) d t=\sum_{1}^{\infty} \alpha_{n} \phi(n) .
$$

Replacing $\phi(x)$ by $\phi(x / k) / k$ in this relation proves (3). Theorem 1 guarantees (4).

Turning now to the general case, it is clearly possible to write $\phi(x)=\phi_{1}(x)+\phi_{2}(x)$ where $\phi_{1}(x)$ and $\Phi_{2}(x)=\phi_{2}(1 / x) / x$ satisfy Conditions B. Thus

$$
F_{2}(x)=\sum_{1}^{\infty} \frac{\alpha_{n}}{n} \Phi_{2}\left(\frac{x}{n}\right)=\sum_{1}^{\infty} \frac{\alpha_{n}}{x} \phi_{2}\left(\frac{n}{x}\right)=g_{2}(x) .
$$


Likewise $G_{2}(x)=f_{2}(x)$. Thus $f(x)=f_{1}(x)+G_{2}(x)$ and $g(x)=g_{1}(x)$ $+F_{2}(x)$, so the validity of (3) is guaranteed by the validity of (3) and (4) in the special case. The same is true of (4).

TheOREM 3. Let $\phi(z)$ be analytic at interior points of the sector $-\Delta \leqq \arg z \leqq \Delta$ for some $\Delta>0$, and in this sector suppose $|\phi(z)|$ $=O\left(|z|^{k}+|z|^{-1-k}\right), 0 \leqq k<1$. Then:

(a) $\phi(x)$ satisfies the conditions of Theorem 2.

(b) The functions $f(z)$ and $g(z)$ satisfy the same conditions as $\phi(z)$ in the sector $-\delta \leqq \arg z \leqq \delta<\Delta$.

(c) If $k=0$ and $\phi(x)=o\left(1+|x|^{-1}\right)$ at 0 and $\infty$, then $f(x)$ and $g(x)$ satisfy the same relation.

(d) In case (c) the series and integrals converge without recourse to summability.

Proof. Let $z$ be a point in the sector $\delta$; then by Cauchy's integral formula for $\phi^{\prime \prime}(z)$ with a circular contour of radius proportional to $|z|$, it follows that $\left|\phi^{\prime \prime}(z)\right|=O\left(|z|^{k-2}+|z|^{-3-k}\right)$. Thus $\int_{1}^{\infty}\left|\phi^{\prime \prime}(x)\right| d x$ $<\infty$, so $\phi^{\prime}(x)$ is of bounded variation in the interval $(1, \infty)$. Similarly, Cauchy's formula for $\phi^{\prime}(z)$ shows that $\phi^{\prime}(x)$ vanishes at $\infty$. Clearly $\Phi(z)$ satisfies the same conditions as $\phi(z)$, so (a) is proved.

Formula (14) may be modified so as to hold for functions which do not vanish at 0 . It is convenient to let $\rho(t)=1 / 2-\dot{\sigma}(t)$. Then it is easy to see that for $z$ in the sector $\delta$

$$
g(z)=\frac{1}{2 z} \phi\left(\frac{1}{z}\right)+\frac{1}{z^{3}} \int_{1}^{\infty} \phi^{\prime \prime}\left(\frac{t}{z}\right) \rho(t) d t .
$$

Introducing the bound for $\phi^{\prime \prime}$ gives

$$
|g(z)| \leqq \frac{1}{2|z|}\left|\phi\left(\frac{1}{z}\right)\right|+O\left(\frac{|z|^{-1-k}}{1-k}+\frac{|z|^{k}}{2+k}\right) .
$$

Thus $|g(z)|=O\left(|z|^{k}+|z|^{-1-k}\right)$. The uniform convergence of (15) guarantees that $g(z)$ is analytic at interior points of the sector $\delta$. Similar considerations apply to $f(z)$, so (b) is proved.

From (15) it is easy to see that if $j$ is an integer of the form $4 n+1$

$$
g(z)=\sum_{1}^{i} \frac{\alpha_{n}}{z} \phi\left(\frac{n}{z}\right)-\frac{1}{2 z} \phi\left(\frac{j}{z}\right)+\frac{1}{z^{3}} \int_{j}^{\infty} \phi^{\prime \prime}\left(\frac{t}{z}\right) \rho(t) d t .
$$

But if $k=0,\left|\phi^{\prime \prime}(z)\right|=O\left(1+|z|^{-1}\right)$, so

$$
|g(x)| \leqq \sum_{1}^{j} \frac{1}{|x|}\left|\phi\left(\frac{n}{x}\right)\right|+o\left(\frac{|x|^{-1}}{j}+\frac{1}{2 j^{2}}\right) .
$$


By choosing $j$ sufficiently large, the last two terms may be made as small as desired for all $x \geqq 1$. By the assumption in (c) it is clear that the series vanishes as $x \rightarrow \infty$. Thus $g(x)$ vanishes at $\infty$. The same inequality shows that $x g(x)$ vanishes at 0 . A similar argument applies to $f(x)$, so part (c) is proved.

It may be seen from (16) that since $\phi(x) \rightarrow 0$, the series for $g(x)$ converges in the ordinary sense. An integration by parts similar to that used in deriving (5) shows that because $f(k)$ and $g(k)$ vanish at $\infty$, the integrals converge in the ordinary sense at $\infty$. Part (b) shows that the integrals (4) and (5) are absolutely convergent at 0.

\section{REFERENCES}

1. R. J. Duffin, Bull. Amer. Math. Soc. vol. 51 (1945) pp. 447-455.

2. S. Bochner, Vorlesungen iber Fouriersche Integrale, Leipzig, 1932, pp. 110 and 224.

Carnegie Institute of Technology 